\title{
Prevalência da colonização pelo estreptococo do grupo B em gestantes atendidas em ambulatório de pré-natal
}

\author{
Prevalence of group B Streptococcus in pregnant women from a prenatal care center \\ Adriane Pogere ${ }^{1}$, Cássia Maria Zoccoli ${ }^{2}$, Nina Reiko Tobouti ${ }^{3}$, Paulo Fontoura Freitas ${ }^{4}$, \\ Armando José d'Acampora ${ }^{5}$, João Nilson Zunino ${ }^{6}$
}

\section{RESUMO}

Objetivo: verificar a prevalência de estreptococo do grupo B (EGB) em gestantes no terceiro trimestre da gravidez e explorar os fatores potencialmente associados à colonização. Métodos: uma amostra de 273 gestantes no terceiro trimestre da gravidez, provenientes do ambulatório de pré-natal do Hospital Universitário do Sul do Brasil, foi investigada. Culturas de amostra vaginal e anorretal foram obtidas e inoculadas em meio seletivo de Todd-Hewitt suplementado com $10 \mu \mathrm{g} / \mathrm{ml} \mathrm{de}$ colistina e $15 \mu \mathrm{g} / \mathrm{ml}$ de ácido nalidíxico e posteriormente subcultivadas em ágar sangue de carneiro desfibrinado. Todas as colônias suspeitas foram submetidas ao teste de aglutinação para deteç̧ão do antígeno específico do grupo B. O teste de Camp foi utilizado para identificação do EGB das variedades não hemolíticas. Analisaram-se também os dados demográficos, socioeconômicos, reprodutivos e clínico-obstétricos. A razão de prevalência (RP) foi utilizada como medida de risco. Considerou-se como significante o intervalo de confiança no nível de $95 \%(\alpha=0,05)$. Resultados: a prevalência de colonização pelo EGB foi de 21,6\% (59), sendo que 9,9\% (27) das gestantes tiveram positividade em ambos os sítios, $6,95 \%$ (19) foram positivas somente no sítio vaginal e 4,75\% (13) da amostra tiveram positividade apenas no sítio anal. A prevalência de EGB foi ligeiramente mais alta nas gestantes com idade inferior a 20 anos, naquelas com menor escolaridade e nas gestantes primíparas, e o dobro entre aquelas que não relataram aborto espontâneo, porém sem significância estatística. Não foi encontrada diferença na prevalência de EGB de acordo com história de doenças sexualmente transmissíveis e tabagismo. Quando os dados foram analisados conjuntamente, os fatores detectados como potencialmente associados à colonização pelo EGB foram: primíparas com mais de 30 anos $(\mathrm{RP}=1,55)$ e mulheres com mais de um parceiro sexual e freqüência de atividade sexual aumentada $(55,6$ vs $20,5 \% ; \mathrm{p}<0,05)$. Conclusão: confirma-se a necessidade rotineira de cultura para EGB em ambos os sítios (vaginal e anal) de todas as gestantes no terceiro trimestre de gestação.

PALAVRAS-CHAVE: Streptococcus agalactiae; Gravidez; Prevalência; Colonização

\section{ABSTRACT}

Purpose: to determine the prevalence of group B Streptococcus (GBS) in pregnant women in the third trimester of pregnancy and explore the factors potentially associated with colonization. Methods: a sample of 273 pregnant women in the third trimester of pregnancy, from the prenatal care center in Southern Brasil, was investigated. Vaginal and anorectal samples were collected and innoculated in Todd-Hewitt selective broth supplemented with $10 \mu \mathrm{g} / \mathrm{mL}$ colistin and $15 \mu \mathrm{g} / \mathrm{mL}$ nalidixic acid and afterwards cultured on defibrinated sheep blood agar plates. All suspected colonies were submitted to the agglutination test for detection of the specific group B antigen. The Camp test was used for GBS identification in non-hemolytic varieties. Demographic, socioeconomic, reproductive, and clinico-obstetric data were also analyzed. Prevalence ratio (PR) was used as risk measurement. Confidence interval was considered significant at the level of $95 \%(\alpha=0.05)$. Results: prevalence of Streptococcus (GBS) colonization amounted to $21.6 \%$ (59), 9.9\% (27) of pregnant women showing positivity in both sites; vaginal site colonization was found in $6.95 \%$ (19) of the women and $4.75 \%$ (13) of the samples showed positivity only in the anal site. GBS prevalence was slightly higher in pregnant women under 20 years, in those with less schooling and in primiparae and was twice as high among those who did not reported spontaneous abortion, but with no statistical significance.

Trabalho desenvolvido na Universidade Federal de Santa Catarina, Grupo de Pesquisa TOCE

1 Ginecologista e Obstetra do Hospital Universitário - Universidade Federal de Santa Catarina

2 Bioquímica e Gerente do Setor de Microbiologia do Laboratório Médico Santa Luzia de Florianópolis

3 Bioquímica e Gerente de Área de Atendimento Hospitalar do Laboratório Médico Santa Luzia de Florianópolis

4 Médico Epidemiologista da Universidade Federal de Santa Catarina

5 Professor Adjunto do Departamento de Clinica Cirúrgica da Universidade Federal de Santa Catarina - Líder do Grupo de Pesquisa TOCE/UFSC

6 Médico Patologista Clínico, Diretor do Laboratório Médico Santa Luzia de Florianópolis

Correspondência: Adriane Pogere

Rua Almirante Lamego, 910 / 804-A - 88015-650 - Florianópolis - SC - fone: (48) 9980-6051 - e-mail: apogere@intercorp.com.br

Recebido em: 4/3/2005 Aceito com modificações em: 16/5/2005

Rev Bras Ginecol Obstet. 2005; 27(4): 174-80 
No difference was found in GBS prevalence according to the history of sexually transmitted diseases and tabagism. When analyzed together, the factors detected as potentially associated with colonization by GBS were: primiparae over 30 years $(\mathrm{PR}=1.55)$ and women with more than one sexual partner and increased frequency of sexual activity $(\mathrm{p}<0.05)(55,6 v s 20.5 \%$; $\mathrm{p}<0.05$ ). No difference regarding prevalence was found to exist in relation to the history of sexually transmitted diseases, previous spontaneous abortion and tabagism. Conclusion: these results confirm the need for routine collection for GBS culture from both sites (vaginal and anal) in all pregnant women in the third trimester of pregnancy.

KEYWORDS: Streptococcus agalactiae; Pregnancy; Prevalence; Colonization

\section{Introdução}

O estreptococo do grupo B (EGB) ou Streptococcus agalactiae é encontrado na mulher como saprófita vaginal ${ }^{1}$, é incomum em crianças, podendo ser encontrado na adolescência tardia. A colonização pelo EGB pode ser transitória, crônica ou intermitente e tem sido isolado em culturas do trato genital e/ou gastrointestinal baixo em 10$40 \%$ das mulheres grávidas ${ }^{2}$. O trato gastrointestinal é o mais provável reservatório do EGB, em humanos. A partir do trato digestivo baixo a bactéria coloniza o trato genital e, menos freqüentemente, o trato urinário ${ }^{3,4}$.

O EGB é a principal causa de sepse e meningite em recém-nascidos, é causa freqüente de pneumonia e é mais comum que outras doenças bem conhecidas, como rubéola, sífilis e espinha bífida ${ }^{1}$. Pode ser responsável pela infecção no organismo materno, comprometendo a evolução da gestação, provocando abortamento, infecção urinária, prematuridade, corioamnionite e endometrite puerperal $^{2,5}$, que podem ser prevenidos pela identificação e tratamento das gestantes colonizadas.

Cerca de 50 a $75 \%$ dos recém-nascidos expostos ao EGB intravaginal tornam-se colonizados e 1 a $2 \%$ de todos recém-nascidos de mães portadoras irão desenvolver doença invasiva de início precoce $^{6}$. Apesar de os bebês prematuros terem um alto risco para adquirir a doença, metade dos casos de sepse ocorrem em bebês a termo ${ }^{6}$. Seqüelas neurológicas ocorrem em cerca de 15 a $30 \%$ dos sobreviventes de meningite ${ }^{6,7}$.

A infecção sistêmica por EGB apresenta duas formas de manifestação clínica no período neonatal: a doença de início precoce e a de início tardio, de acordo com Baker e Morven, em 1973³. A infecção de início precoce, que ocorre em dois terços dos casos, manifesta-se nas primeiras 24 horas de vida em 60 a $80 \%$ dos casos. As formas de manifestação mais comuns são a sepse com bacteriemia sem localização da infecção (25 a 40\% dos casos), a pneumonia ( 35 a $55 \%$ ) e, menos comumente, a meningite $(5 \text { a } 15 \%)^{3,7}$. A taxa de letalidade já alcançou até $50 \%$. Ao redor de 1990 esta taxa já havia diminuído para 4\%, devido aos avanços quanto à profilaxia e cuidados neonatais ${ }^{1}$. A doença de início tardio que ocorre em um terço dos casos manifesta-se entre 7 dias e 12 semanas de idade, com média de 27 dias, e pode ser conseqüência de transmissão vertical, em menor proporção, ou nosocomial, a partir do pessoal e/ou outros recém-nascidos colonizados. Apresenta-se como meningite em 30\% dos casos. Doença localizada em tecidos moles, ossos e articulações também são manifestações encontradas quase que exclusivamente neste periodo. As taxas de letalidade são baixas ( 2 a $6 \%$ dos casos), mas 25 a $50 \%$ dos sobreviventes têm seqüelas neurológicas permanentes ${ }^{4}$.

Alguns fatores aumentam o risco de infecção de início precoce no recém-nascido e o mais evidente deles é a colonização materna pelo EGB no momento do parto ${ }^{1}$. Diante disso, medidas profiláticas como antibioticoterapia durante o trabalho de parto têm sido incentivadas em nivel internacional, com o intuito de diminuir a incidência de sepse neonatal e suas possiveis conseqüências ao recém-nascido.

Este microrganismo, que desencadeia toda esta gama de morbidades, permaneceu desconhecido até 1970, quando ocorreu aumento importante da incidência de septicemia e meningite em neonatos causados pelo EGB ${ }^{1}$. Muitos estudos já documentaram maior acurácia do método baseado em cultura, com identificação das mulheres colonizadas no momento do parto, para prevenção da septicemia neonatal ${ }^{8,9}$. Este método pode alcançar eficácia máxima quando alguns cuidados são observados: época gestacional da realização da cultura, sítios anatômicos para coleta da amostra e métodos microbiológicos precisos para cultura e detecção do $\mathrm{EGB}^{10}$.

A idade gestacional preconizada para realização da cultura para identificar portadoras do EGB é 35-37 semanas, porque é o período no qual se demonstrou melhor sensibilidade e especificidade para detecção de mulheres que permanecem colonizadas por ocasião do parto ${ }^{1,11}$. A coleta do material para cultura tem se mostrado mais eficaz quando realizada no terço inferior do trato genital 
(intróito vaginal) e região anal (dentro do esfincter anal). Estudos não têm concluído pela recomendação de culturas cervicais, da região vaginal somente e nem o uso de espéculo vaginal para a coleta ${ }^{1}$.

$\mathrm{O}$ uso de meios seletivos contendo agentes antimicrobianos para inibir o crescimento de outros microrganismos são essenciais porque podem aumentar em até $50 \%$ a positividade das culturas ${ }^{3}$.

Variações na prevalência da colonização pelo EGB, encontradas na literatura, podem ser atribuidas tanto a diferenças nas características das populações (estudadas como idade, paridade, nível socioeconômico e localização geográfica) quanto às diferenças nos métodos de cultura utilizados. A epidemiologia da ocorrência do EGB, tanto na gestante como no neonato, tem sido muito estudada nos EUA e Europa, mas não na América Latina. A escassez de informação a respeito de sua ocorrência em nosso meio pode ser vista como responsável, pelo menos em parte, pela pouca atenção dada pelos órgãos responsáveis pela vigilância e prevenção tanto no que se refere à investigação das gestantes durante o pré-natal, quanto à profilaxia correta das mulheres colonizadas no momento do parto, levando a investigar a prevalência de EGB, em gestantes assintomáticas, atendidas em ambulatório de Hospital Universitário do Sul do Brasil.

\section{Pacientes e Métodos}

Trata-se de estudo transversal e prospectivo (inquérito de prevalência). A população estudada constituiu-se de todas as grávidas no terceiro trimestre de gestação atendidas no ambulatório de prénatal do Hospital Universitário da Universidade Federal de Santa Catarina, na cidade de Florianópolis, no período de primeiro de outubro de 2002 a 30 de agosto de 2003, totalizando 273 gestantes.

As gestantes, para participarem da pesquisa, foram devidamente informadas sobre os objetivos e procedimentos antes de aceitarem participar e de assinarem o consentimento livre e informado, e o estudo foi aprovado pelo Comitê de Ética com Seres Humanos da Universidade Federal de Santa Catarina, em abril de 2002.

Os critérios de inclusão utilizados foram: gestantes de 35 ou mais semanas de gestação que foram atendidas no ambulatório de pré-natal do Hospital Universitário da Universidade Federal de Santa Catarina e que aceitaram participar da pesquisa. $\mathrm{Ne}-$ nhuma das pacientes comunicadas recusou-se a participar. Foram excluídas as grávidas que haviam sido submetidas a exame ginecológico, no mesmo dia e anteriormente a coleta, ou que haviam utilizado antibióticos há pelo menos duas semanas.
A cada gestante foi aplicado um questionário com perguntas fechadas e pré-codificadas a respeito de dados demográficos, socioeconômicos, reprodutivos e clínico-obstétricos. Dados sobre a gestação atual também foram obtidos do prontuário médico da paciente. O número de parceiros sexuais se refere àquelas relações ocorridas no período de um ano antes da gestação até o momento da entrevista. Foram consideradas como primiparas aquelas mulheres com um parto e multiparas com dois partos ou mais, no momento da entrevista. A idade gestacional foi avaliada pela data da última menstruação e/ou pela ultrasonografia mais precoce. A entrevista e a coleta do material de todas as mulheres foram realizadas pela autora, não tendo sido permitida a presença de acompanhantes durante estes procedimentos, a fim de evitar omissão de dados.

Os sitios de coleta para cultura do EGB foram: a) cultura vaginal, obtida com a introdução de um swab estéril alginatado através do intróito vaginal e coleta de amostras do terço distal da vagina, sem uso de espéculo; b) cultura anorretal, obtida com um segundo swab estéril, com introdução através do orificio anal para coleta de amostras da parede distal do reto. Cada um dos dois swabs foi inoculado em tubo de ensaio individual, devidamente rotulado, contendo meio de cultura seletivo, caldo de Todd-Hewitt suplementado com $10 \mu \mathrm{g} / \mathrm{mL}$ de colistina e $15 \mu \mathrm{g} / \mathrm{mL}$ de ácido nalidixico. Este meio foi previamente preparado e armazenado pelo setor de microbiologia do Laboratório Médico Santa Luzia, na cidade de Florianópolis. O material colhido foi enviado novamente a este laboratório para cultura e análise dos resultados.

Os caldos seletivos foram incubados por 18 a 24 horas, a $35^{\circ} \mathrm{C}$ e subcultivados em ágar sangue de carneiro desfibrinado. Após 24 horas de incubação as placas foram inspecionadas e as colônias sugestivas de EGB ( $\beta$-hemolíticas ou não, gram-positivas e catalase-negativas) foram então identificadas. Quando o estreptococo $\beta$-hemolitico não foi identificado após 18 a 24 horas de incubação, em placa de ágar sangue de carneiro, as mesmas foram reincubadas por mais 24 horas, e a leitura final efetuada em 48 horas. Todas as colônias suspeitas foram subcultivadas e submetidas ao teste de aglutinação para detecção do antígeno específico do grupo B. O teste de Camp foi utilizado para identificação do EGB, principalmente as variedades não hemoliticas. Este teste é muito utilizado para identificação do EGB e consiste na produção de uma proteína extracelular difusível (fator Camp) pelo EGB, que reage, sinergicamente com uma beta-lisina do Staphylococcus aureus cau- 
sando lise dos eritrócitos. Um teste positivo aparece como uma zona de hemólise, em forma de "ponta de flecha", na área em que a beta-lisina estafilocócica e o fator Camp estão difundidos.

Os dados coletados foram digitados a partir do protocolo e um questionário eletrônico foi criado utilizando o programa Epidata 3.0. A razão de prevalência (RP) foi utilizada como medida de risco na comparação entre as ocorrências do desfecho. Para aquelas variáveis apresentando associação com o desfecho (RP>1) são apresentados os intervalos de confiança no nível de $95 \%(\alpha=0,05)$. Nesta fase da análise foi utilizado o programa Epi-Info 6.04.

\section{Resultados}

Das 273 gestantes incluídas, 21,6\% (59) tiveram cultura positiva para EGB, ao passo que $78,4 \%$ (214) tiveram cultura negativa.

Ao estratificar a amostra de acordo com o sítio anatômico da coleta encontrou-se diferença na positividade de acordo com o local, como mostrado na Tabela 1. Como observado, 27 mulheres $(9,9 \%)$ foram positivas em ambos os sitios, 19 $(6,9 \%)$ no sítio vaginal e $13(4,7 \%)$ no anal.

A prevalência de EGB foi ligeiramente maior nas gestantes com idade inferior a 20 anos, naquelas com menor escolaridade e nas gestantes primíparas, e o dobro entre aquelas que não relataram aborto espontâneo $(\mathrm{RP}=2,08)$, ainda que estas diferenças não tenham sido estatisticamente significantes. Não foi encontrada diferença na prevalência de EGB de acordo com tabagismo (Tabela 2).

Ao avaliar a prevalência de EGB de acordo com o grupo etário estratificado para os efeitos de paridade, ou seja, quando idade e paridade foram avaliadas conjuntamente, verifica-se que o risco (RP) de colonização entre as primíparas cresce de acordo com um aumento no grupo etário, ao passo que entre as multiparas o risco toma o sentido inverso. A prevalência de EGB encontrada foi $55 \%$ maior $(\mathrm{RP}=1,5)$ entre as primiparas mais velhas

Tabela 1 - Distribuição das gestantes de acordo com o resultado da cultura para estreptococo do grupo $B$ (EGB) e sítio de colonização.

\begin{tabular}{lcccc}
\hline Sítio anatômico & \multicolumn{2}{c}{ EGB (+) } & \multicolumn{2}{c}{ EGB ( - ) } \\
& $\mathbf{n}$ & $\%$ & $\mathbf{n}$ & $\%$ \\
\hline Vaginal & 19 & 6,9 & 254 & 93,0 \\
Anal & 13 & 4,7 & 260 & 95,2 \\
Vaginal + anal & 27 & 9,9 & 246 & 90,1 \\
Total & 59 & 21,6 & 214 & 78,4 \\
\hline
\end{tabular}

comparadas às mais jovens, ao passo que entre as multiparas o efeito se inverte, com o risco $80 \%$ maior $(\mathrm{RP}=1,80)$ entre as mais jovens comparadas às mais velhas (Figura 1).

Tabela 2 - Prevalência de estreptococo do grupo B (EGB) de acordo com os fatores de risco potencialmente associados a sua ocorrência.

\begin{tabular}{lcccc}
\hline & Grupo & \multicolumn{2}{c}{ EGB(+) } & IC 95\% \\
& \multicolumn{4}{c}{ n (+)/n sob risco \% } \\
\hline Idade & <de 20 anos & $10 / 43$ & 23,3 & $11,8-38,6$ \\
& 20 a 29 anos & $30 / 139$ & 21,6 & $15,1-29,4$ \\
& $>$ de 30 anos & $19 / 91$ & 20,9 & $13,1-30,7$ \\
Escolaridade & 1 a 8 anos & $32 / 138$ & 23,2 & $16,4-31,1$ \\
& 9 anos ou mais & $27 / 13$ & 20,0 & $13,7-27,9$ \\
Paridade & Primípara & $29 / 117$ & 24,8 & $17,3-33,6$ \\
& Multípara & $30 / 156$ & 19,2 & $13,4-26,3$ \\
Aborto espontâneo & Sim & $6 / 52$ & 11,5 & $4,4-23,4$ \\
pregresso & Não & $53 / 221$ & 24,0 & $18,5-30,2$ \\
Fumante & Sim & $6 / 28$ & 21,4 & $8,3-41$ \\
& Não & $53 / 245$ & 21,6 & $16,6-27,3$ \\
\hline
\end{tabular}

IC: Intervalo de confiança.

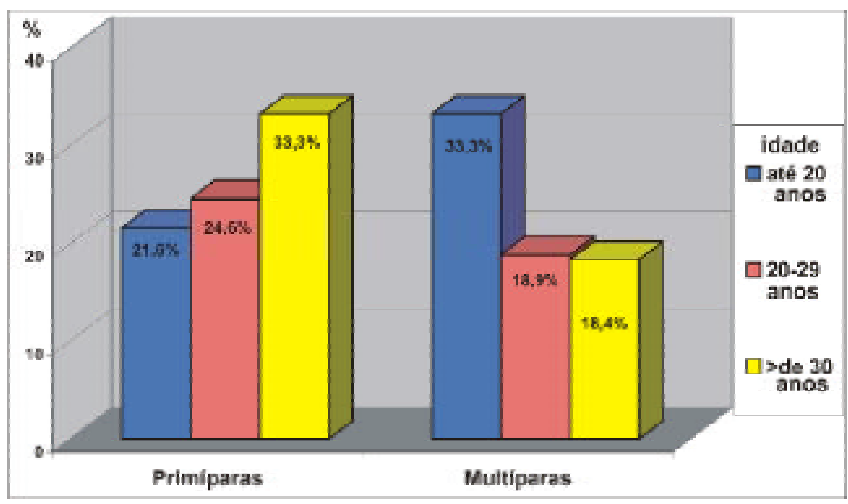

Figura 1 - Prevalência de estreptococo do grupo B (EGB), levando em conta os efeitos conjuntos da idade e paridade.

Foi encontrada prevalência de colonização por EGB 50\% maior entre as mulheres com maior freqüência de relações sexuais $(28,2$ vs $18,6 \%)$, beirando a significância estatística no nível de $95 \%$ de confiança $(p=0,07)$. A prevalência de EGB foi também 1,5 vezes maior entre as mulheres com mais de um parceiro sexual (33,3 vs $20,8 \%$ ), apesar de esta diferença não ter sido estatisticamente significante.

Os resultados encontrados neste estudo mostram positividade aumentada para o EGB nas mulheres com relações sexuais mais freqüentes (três ou mais por semana) e naquelas com dois ou mais 
parceiros (no período de um ano anterior à gravidez até o momento da entrevista), apesar de não se evidenciar significância. Mas, quando selecionado um grupo de maior risco para colonização pelo EGB, que consistiu das mulheres com mais de três relações sexuais por semana e mais de um parceiro sexual, foi encontrada prevalência de positividade para EGB significantemente maior no grupo de risco para atividade sexual $(55,6 \%)$, ou seja, quase o triplo da prevalência, de $20,5 \%$, encontrada para o restante da amostra $(\mathrm{p}<0,05)$.

No presente estudo, em 10,6\% da amostra as gestantes entrevistadas relataram doença sexualmente transmissivel prévia (DST) ou durante a atual gestação. Entretanto, nenhuma diferença foi encontrada ao se comparar aquelas mulheres com e sem história de DST $(21,4$ vs $21,6 \%)$, demonstrando que este não foi fator importante na via de transmissão do EGB.

\section{Discussão}

A prevalência da colonização por EGB varia bastante de acordo com a localização geográfica, inclusive com flutuações regionais. Aproximadamente 10 a $40 \%$ das gestantes são colonizadas na vagina ou no reto ${ }^{3,12}$. Sabendo-se também que a prevalência do EGB é relacionada a outras variáveis como: idade, paridade, localização geográfica, métodos de cultura, nível sócio-econômico ${ }^{13}$, torna-se importante detectar os fatores que mais provavelmente levarão ao desfecho, bem como a prevalência nas diferentes regiões para permitir que medidas adequadas de controle e prevenção sejam implantadas. É bem documentado que alguns fatores como idade gestacional na época da realização da triagem para colonização por EGB, sítios anatômicos pesquisados e métodos microbiológicos utilizados melhoram a precisão dos resulta$\operatorname{dos}^{10}$.

Uma revisão de 1998 sobre a prevalência de colonização por EGB em países em desenvolvimento, foram analisados os baixos índices de infecção neonatal por EGB nestas regiões. Quando se compararou aqueles estudos considerados adequados (em que meio seletivo foi utilizado e dentre os sítios coletados a vagina estava incluída), foram observadas as seguintes taxas de colonização em mulheres: Índia/Paquistão, 12\%; Ásia/Pacífico, 19\% ; África, 19\%; norte da África, 22\% ; Américas, $14 \%$. Foi sugerido, nesta revisão, que as taxas de colonização podem ser subestimadas em muitos estudos devido às inadequadas técnicas de cultura empregadas. Neste contexto, observa-se que independentemente do status de desenvolvimento socioeconômico, a discrepância nas taxas de colonização relatadas em diferentes meios tem relação direta com as técnicas específicas de coleta e cultura empregadas, e assim estas diferenças devem obrigatoriamente ser levadas em conta ao comparar resultados para diferentes países, regiões ou serviços. A principal falha encontrada em muitas regiões, inclusive em nosso meio, é a não utilização de meios seletivos e coleta apenas do sítio vaginal, excluindo-se o anal.

No Brasil a taxa de colonização varia de 15 a $25 \%{ }^{15-17}$. Esta variação é devida aos diferentes métodos utilizados. A menor porcentagem foi observada em estudo no qual os sítios pesquisados foram o vaginal e periuretral e o meio seletivo não foi utilizado ${ }^{15}$.

Em 2002, num estudo realizado na cidade de São Paulo sobre incidência de sepse neonatal por EGB, foi relatado $13 \%$ de positividade para esta bactéria nas gestantes da instituição, mas somente amostra vaginal foi realizada e o método de cultura utilizado não foi especificado. Nesta mesma pesquisa, a incidência de infecção neonatal precoce foi de 0,39 casos por 1000 nascidos vivos, taxa menor que a observada em países desenvolvidos, sendo que em $30 \%$ dos casos não foi possível detectar o agente etiológico ${ }^{18}$. Por outro lado, estudo realizado em 1997, em Ribeirão Preto no Estado de São Paulo, demonstrou que o EGB foi a bactéria mais prevalente nos casos de sepse neonatal, estando presente em $37,8 \%$ dos casos de infecção confirmada $^{7}$. Em 2001, em Porto Alegre no Rio Grande do Sul, foi demonstrado que o EGB foi um dos patógenos mais importantes na etiologia de sepse de início precoce em neonatos, com incidência de $1 / 1000$ nascidos vivos ${ }^{19}$.

A prevalência de colonização deste estudo $(21,6 \%)$ é similar às encontradas no Rio de Janeiro $(25,6 \%)^{17}$ e em Florianópolis $(25,2 \%)^{16}$, trabalhos com metodologias semelhantes e julgadas adequadas pelos autores. Diante disso, parece que o meio seletivo é um dos fatores que leva ao diagnóstico de certeza da infecção pelo EGB e, quando utilizado, as taxas de prevalência de colonização são muito semelhantes em todos os trabalhos encontrados, independente da origem.

Observa-se aumento de 5 a $27 \%$ da detecção do EGB quando os sítios vaginal e anal são pesquisados $^{12}$. No presente estudo, tomando em conta que $14,7 \%$ (13 mulheres) tiveram positividade ao EGB somente no sítio anal, $4,7 \%$ do total da amostra $(13 / 273)$ não seria diagnosticada, caso a cultura fosse realizada somente no sítio vaginal. Vale salientar novamente que, em muitas regiões, o sítio anal não é pesquisado, subestimando desta 
maneira as verdadeiras taxas de prevalência. Esta subestimativa, como já citado anteriormente, pode explicar em grande parte a ausência de medidas de vigilância em nosso meio, entre elas aquelas dirigidas à prevenção da sepse neonatal pelo EGB com graves conseqüências para o recém-nascido.

Vários fatores de risco, citados na literatura, têm sido avaliados, por sua possivel influencia na colonização genital das mulheres. Mães adolescentes e com menor paridade têm sido apontadas por grande número de estudos como grupos de risco para a colonização pelo $\mathrm{EGB}^{3}$. Entretanto, existe discordância a respeito do efeito destas duas variáveis sobre a ocorrência de colonização. Orret $^{20}$, em 2003, encontrou taxas significantemente maiores entre as multiparas do que entre as primíparas. De acordo com Regan et al. ${ }^{13}$, quando a paridade foi controlada em relação à idade, houve diminuição do risco de colonização com o aumento da paridade e aumento do risco com o aumento da idade. De acordo com este estudo, mulheres mais velhas com baixa paridade têm maior risco de serem colonizadas por EGB.

Este estudo mostra resultados semelhantes aos de outros autores quanto à existência de correlação negativa entre a prevalência de EGB, idade e paridade, analisadas separadamente, porém quando da estratificação da amostra levando em conta os efeitos conjuntos das duas variáveis, encontrou positividade para o EGB 55\% maior entre as primiparas com mais de 30 anos comparadas àquelas primíparas mais jovens, e sentido inverso no efeito entre as multíparas, com risco $80 \%$ maior entre as multiparas jovens comparadas às mais velhas, chegando assim à mesma conclusão que Regan et al. ${ }^{13}$.

Segundo Baker e Morven ${ }^{3}$, estado civil, freqüência do intercurso sexual e número de parceiros sexuais não influenciaram na prevalência da colonização genital. Mas de acordo com Regan et al. ${ }^{13}$, não houve aumento na incidência de colonização entre aquelas mulheres com parceiro fixo, desde o ano anterior à gravidez, mesmo com relações sexuais freqüentes, nem entre aquelas com mais de um parceiro e com relações sexuais ocasionais. Mas, quando ambas as variáveis foram associadas, múltiplos parceiros e intercurso sexual freqüente, foi observado aumento da prevalência de colonização. Este estudo chegou aos mesmos resultados de Regan et al. ${ }^{13}$, demonstrando significância estatística.

Não houve, conforme os resultados obtidos, em concordância com a maioria das pesquisas, como correlacionar DST e colonização pelo $\mathrm{EGB}^{10,21}$. No sentido inverso, estão os achados de maior prevalência em mulheres com freqüência maior de relações sexuais por semana e mais de um parceiro sexual, inclusive com resultados estatisticamente significativos quando estas duas variáveis foram comparadas em relação ao restante da amostra, como já foi citado anteriormente, indicando a promiscuidade como um fator importante na colonização. Diante disso, outros estudos ainda se mostram necessários, para poder afirmar a respeito do modo de transmissão desta bactéria.

Embora a colonização por EGB seja citada na literatura como causa de abortamento entre as mulheres ${ }^{22}$, este estudo encontrou que a prevalência de EGB foi duas vezes maior nas mulheres sem história de aborto espontâneo prévio.

Alguns autores citam o hábito de fumar como possivel fator de risco para colonização por EGB. Regan et al. ${ }^{13}$, descrevem que mulheres que nunca fumaram possuem discreto aumento no risco de colonização quando comparadas com as fumantes. Contrariamente a estes dados, Terry et al. ${ }^{23}$, num estudo com 543 mulheres, encontraram taxa de prevalência entre as fumantes de $33,1 \%$ quando comparadas às não-fumantes $(16,4 \%)$, porém sem significância estatística. Neste estudo, apesar de somente 10,3\% das mulheres analisadas possuírem o hábito de fumar, nenhuma diferença na prevalência foi encontrada, de acordo com esta variável.

Embora alguns fatores associados à colonização para o EGB durante a gestação pudessem ser identificados, isto não nos permite selecionar um grupo de mulheres com alta probabilidade de serem portadoras, apenas baseados nos fatores de risco (físicos e da história clínica), confirmando ainda mais a necessidade de investigar todas as gestantes no terceiro trimestre. Os resultados em relação à prevalência do EGB buscam subsidiar a necessidade de informação, dirigida a elaborar medidas de prevenção baseadas em evidências.

\section{Referências}

1. Schrag S, Gorwitz R, Fultz-Butts K, Schuchat A. Prevention of perinatal group $B$ streptococcal disease. Revised guidelines from CDC. MMWR Recomm Rep. 2002;51(RR-11):1-22.

2. Gibbs RS, Schrag S, Schuchat A. Perinatal infections due to group B streptococci. Obstet Gynecol. 2004;104(5 Pt 1):1062-76.

3. Baker CJ, Morven ES. Group B streptococcal infections. In: Remington JS, Klein JO, editors. Infectious disease of the fetus and the newborn infant. $5^{\text {th }}$ ed. Philadelphia: Saunders; 1998. p. 1091156. 
4. Turow J, Spitzer AR. Group B streptococcal infection early onset disease controversies in prevention guidelines, and management strategies for the neonate. Clin Pediatr (Phila). 2000;39(6):317-26.

5. Ovalle Salas A, Gómez M. R, Martinez Tagle MA, Aspillaga Manterola C, Dolz C. S. Infección vaginal y tratamiento del streptococcus grupo B en embarazadas con factores universales de riesgo de infección: resultados neonatales y factores de riesgo de infección neonatal. Rev Chil Obstet Ginecol. 2002;67(6):465-75.

6. Mercola J. Group B streptococcus. Midwifery Today E-News. 2001 Sep. 12;3(37).

7. Nobre RA. Freqüência de infecção por streptococcus b-hemolítico do grupo B e outras bactérias em recém-nascidos com desconforto respiratório [dissertação]. Ribeirão Preto: Universidade de São Paulo; 1997.

8. Apgar BS, Greenberg G, Yen G. Prevention of group $B$ streptococcal disease in the newborn. Am Fam Physician. 2005;71(5):903-10.

9. Halliday E, Foote K, Dryden M, Heard M, Down R, Ward J. Universal maternal screening for neonatal group B streptococcal disease. Lancet. 2000;356(9239):1407-8.

10. Gilson GJ, Christensen F, Romero H, Bekes K, Silva L, Qualls CR. Prevention of group B streptococcus early-onset neonatal sepsis: comparison of the Center for Disease Control and prevention screening-based protocol to a risk-based protocol in infants at greater than 37 weeks' gestation. J Perinatol. 2000;20(8 Pt 1):491-5.

11. Money DM, Dobson S. The prevention of early-onset neonatal group B streptococcal disease. J Obstet Gynaecol Can. 2004;26(9):826-40.

12. Centers for Disease Control and Prevention (CDC). Laboratory practices for prenatal group B streptococcal screening-seven states, 2003. MMWR Morb Mortal Wkly Rep. 2004;53(23):506-9.

13. Regan JA, Klebanoff MA, Nugent RP. The epidemiology of group B streptococcal colonization in pregnancy. Vaginal Infections and Prematurity Study Group. Obstet Gynecol. 1991;77(4):604-10.
14. Stoll BJ, Schuchat A. Maternal carriage of group B streptococci in developing countries. Pediatr Infect Dis J. 1998;17(6):499-503.

15. Mocelin CO, Carvalho DAF, Brites C, Christofolli $\mathrm{D}$, Mocelin AO, Fracalanzza SEL, et al. Isolamento de Streptococcus agalactiae de gestantes na região Londrina-PR. Rev Bras Ginecol Obstet. 1995;17(9):915-8.

16. Smânia Júnior A, Benchetrit LC, Smânia EFA, Fracalanzza SEL. Isolamento de estreptococos do grupo $\mathrm{B}$, de gestantes e neonatos, em Florianópolis, Santa Catarina. Rev Bras Anal Clin. 1986;18(4):103-8.

17. Benchetrit LC, Fracalanzza SE, Peregrino H, Camelo AA, Sanches LA. Carriage of Streptococcus agalactiae in women and neonates and distribution of serological types: a study in Brazil. J Clin Microbiol. 1982;15(5):787-90.

18. Vaciloto E, Richtmann R, de Paula Fiod Costa H, Kusano EJ, de Almeida MF, Amaro ER. A survey of the incidence of neonatal sepsis by group $B$ Streptococcus during a decade in a Brazilian maternity hospital. Braz J Infect Dis. 2002;6(2):55-62.

19. Miura E, Martin MC. Group B streptococcal neonatal infections in Rio Grande do Sul, Brazil. Rev Inst Med Trop Sao Paulo. 2001;43(5):243-6.

20. Orrett FA. Colonization with Group B streptococci in pregnancy and outcome of infected neonates in Trinidad. Pediatr Int. 2003;45(3):319-23.

21. Collins TS, Calderon M, Gilman RH, Vivar A, Charache P. Group B streptococcal colonization in a developing country: its association with sexually transmitted disease and socioeconomic factors. Am J Trop Med Hyg. 1998;59(4):633-6.

22. McDonald HM, Chambers HM. Intrauterine infection and spontaneous midgestation abortion: is the spectrum of microorganisms similar to that in preterm labor? Infect Dis Obstet Gynecol. 2000;8(56):220-7.

23. Terry RR, Kelly FW, Gauzer C, Jeitler M. Risk factors for maternal colonization with group B betahemolytic streptococci. J Am Osteopath Assoc. 1999;99(11):571-3. 